\title{
Combinations of stroke neurorehabilitation to facilitate motor recovery: perspectives on Hebbian plasticity and homeostatic metaplasticity
}

\author{
Naoyuki Takeuchi* and Shin-Ichi Izumi \\ Department of Physical Medicine and Rehabilitation, Tohoku University Graduate School of Medicine, Sendai, Japan
}

OPEN ACCESS

Edited by:

A. M. Barrett,

Kessler Foundation, USA

Reviewed by:

Friedhelm C. Hummel,

University Medical Center Hamburg-Eppendorf, Germany

Gottfried Schlaug,

Beth Israel Deaconess Medical Center and Harvard Medical School, USA

Ela B. Plow,

Cleveland Clinic Foundation, USA

*Correspondence:

Naoyuki Takeuchi

Department of Physical Medicine and

Rehabilitation, Tohoku University

Graduate School of Medicine, 2-1

Seiryo-cho, Aoba-ku, Sendai

980-8575, Japan

naoyuki@med.hokudai.ac.jp

Received: 30 December 2014 Accepted: 31 May 2015

Published: 23 June 2015

Citation:

Takeuchi N and Izumi S-I (2015)

Combinations of stroke neurorehabilitation to facilitate motor recovery: perspectives on Hebbian plasticity and homeostatic metaplasticity

Front. Hum. Neurosci. 9:349 doi: 10.3389/fnhum.2015.00349
Motor recovery after stroke involves developing new neural connections, acquiring new functions, and compensating for impairments. These processes are related to neural plasticity. Various novel stroke rehabilitation techniques based on basic science and clinical studies of neural plasticity have been developed to aid motor recovery. Current research aims to determine whether using combinations of these techniques can synergistically improve motor recovery. When different stroke neurorehabilitation therapies are combined, the timing of each therapeutic program must be considered to enable optimal neural plasticity. Synchronizing stroke rehabilitation with voluntary neural and/or muscle activity can lead to motor recovery by targeting Hebbian plasticity. This reinforces the neural connections between paretic muscles and the residual motor area. Homeostatic metaplasticity, which stabilizes the activity of neurons and neural circuits, can either augment or reduce the synergic effect depending on the timing of combination therapy and types of neurorehabilitation that are used. Moreover, the possibility that the threshold and degree of induced plasticity can be altered after stroke should be noted. This review focuses on the mechanisms underlying combinations of neurorehabilitation approaches and their future clinical applications. We suggest therapeutic approaches for cortical reorganization and maximal functional gain in patients with stroke, based on the processes of Hebbian plasticity and homeostatic metaplasticity. Few of the possible combinations of stroke neurorehabilitation have been tested experimentally; therefore, further studies are required to determine the appropriate combination for motor recovery.

Keywords: stroke, motor recovery, rehabilitation, Hebbian plasticity, homeostatic metaplasticity

\section{Introduction}

Advances in non-invasive brain imaging technologies have increased our understanding of neural plasticity, which induces the functional and structural changes in the central nervous system after stroke and rehabilitation (Lindenberg et al., 2010a; Rehme et al., 2011; Westlake et al., 2012; De Vico Fallani et al., 2013; Grefkes and Ward, 2014; Liuzzi et al., 2014; Stinear and Byblow, 2014; Schulz et al., 2015). Neural plasticity is the ability of the brain to develop new neuronal connections, acquire new functions, and compensate for impairments (Murphy and Corbett, 2009, 
Dimyan and Cohen, 2011, Takeuchi and Izumi, 2012a). These processes are crucial for motor recovery after stroke. Although various neurorehabilitation strategies are emerging to enhance beneficial plasticity and improve motor recovery after stroke, these interventions show large inter-individual variations in efficacy, due to the heterogeneous mechanisms underlying motor recovery across patients (Langhorne et al., 2011; Pollock et al., 2014; Veerbeek et al., 2014). Therefore, investigations are underway to determine if employing combinations of these techniques can maximize motor recovery by strengthening neural plasticity. Combining protocols that are based on different mechanisms, rather than using each therapy alone, is expected to complement and augment the effects of each therapy to more stably and synergistically facilitate motor recovery.

However, when stroke neurorehabilitation therapies are combined, Hebbian plasticity and homeostatic metaplasticity, as well as ceiling effects, must be taken into account. New or reinforced neural connections induced by Hebbian plasticity is proposed to target plasticity primarily between paretic muscles and residual cortical representations, providing a more focused rehabilitation strategy (Edwardson et al., 2013). The time window for long-term potentiation (LTP)-like plasticity between the cortex and periphery is very narrow and a delay in brain stimulation following movement onset might conversely induce long-term depression (LTD)-like plasticity (Thabit et al., 2010; Massie et al., 2015). Facilitatory priming followed by other facilitatory strategies may diminish the aftereffects of the latter by triggering homeostatic metaplasticity that maintains neural network activity (Abraham, 2008; Jung and Ziemann, 2009; Fricke et al., 2011).

In this review, we first overview the principles of Hebbian plasticity and homeostatic metaplasticity that underlie these synergic effects. We then discuss the different combinations of neurorehabilitation therapy in relation to distinct or simultaneous timing. The concept of priming helps us to understand the interaction between neurorehabilitation therapies may be difficult to perform simultaneously; however, inappropriate timing between interventions that may cause their synergic effect to disappear due to homeostatic metaplasticity. Next, we discuss how simultaneous central or peripheral stimulation contingent on voluntary neural or muscle activity can induce activity-dependent plasticity. We then describe the development of technology that can provide real-time brain activity monitoring that will assist in identifying the narrow time window available to induce activity-dependent plasticity for motor recovery after stroke. Lastly, we discuss the future of combined neurorehabilitation to facilitate the synergy of these processes. Combinations of stroke motor neurorehabilitation techniques are currently being applied clinically; therefore, for future research, it is important to discuss current practices and highlight the gaps in our knowledge.

\section{Hebbian Plasticity and Homeostatic Metaplasticity}

Neural plasticity is tightly regulated and can be significantly affected by the timing of when stroke neurorehabilitation therapies are combined. In this section, we overview activitydependent stimulation targeting Hebbian plasticity and homeostatic metaplasticity, which may influence the timing of therapy with respect to the effectiveness of combined stroke neurorehabilitation.

\section{Activity-Dependent Stimulation Targeting Hebbian Plasticity}

There is much evidence from basic science about the important roles of activity-dependent plasticity after motor learning (Classen et al., 1998; Butefisch et al., 2000; Muellbacher et al., 2001). In the human motor system, it has been well described that activity-dependent plasticity can be artificially induced by brain stimulation in a manner contingent on strict temporal relationships in central or peripheral voluntary activity. This method is based on Hebbian plasticity, in which synaptic plasticity is strengthened when presynaptic and postsynaptic neurons are coincidentally active (Hebb, 1949). In humans, paired associative stimulation (PAS) is the most studied methodology for inducing activity-dependent cortical stimulation (Stefan et al., 2000; Muller-Dahlhaus et al., 2010). PAS is carried out using low-frequency pairing median nerve stimulation at the wrist with delayed transcranial magnetic stimulation (TMS) to the region of the contralateral motor cortex (M1) that represents the thumb (Stefan et al., 2000). This LTP/LTD-like plasticity is dependent on the timing between median nerve stimulation and TMS. LTP-like plasticity is induced when median nerve stimulation precedes TMS by 25 or $2 \mathrm{~ms}$ after the individual N20 latency of the median nerve somatosensory evoked potential, whereas LTD-like plasticity is induced using an intersimulus interval of 10 or $5 \mathrm{~ms}$ before the N20 latency (Wolters et al., 2003; Ziemann et al., 2004; Muller et al., 2007).

In addition to peripheral sensory stimulation, other studies have revealed that activity-dependent cortical stimulation by TMS over M1 synchronizes with voluntary muscle movement. Butefisch et al. studied whether TMS over the M1 synchronously applied with motor training could enhance the encoding of motor memory (Butefisch et al., 2004). Subjects practiced $1 \mathrm{~Hz}$ 30 -min brisk thumb voluntary movements in the opposite direction evoked by TMS over the M1 region that represents the thumb movement. A subthreshold TMS triggered by the muscle activity was applied at $0.1 \mathrm{~Hz}$ to the contralateral or ipsilateral M1 in synchrony with 1 out of every 10 voluntary thumb movements. This study showed that movement-dependent encoding of a motor memory for a trained direction can be enhanced by synchronous TMS at the contralateral M1 and reduced by TMS at the homologous ipsilateral M1. Thabit et al. reported that TMS over M1 paired with $0.2 \mathrm{~Hz} 20$-min thumb voluntary movement can induce changes in the corticospinal excitability and motor behavior (Thabit et al., 2010). When TMS was delivered $50 \mathrm{~ms}$ before movement reaction time, the cortical excitability of the target muscle increased for up to $15 \mathrm{~min}$ after stimulation. This also shortened the target muscle reaction time. However, when TMS was delivered $100 \mathrm{~ms}$ after the reaction time, cortical excitability was decreased. Massie et al. investigated the use of synchronous TMS over M1 during robotic reaching training in the opposite direction evoked by TMS (Massie et al., 2015). TMS was synchronously applied during alternate reaching tasks 
that consisted of three blocks of 160 trials. When TMS was delivered approximately $150 \mathrm{~ms}$ before the reaction time, M1 cortical excitability representing muscles involved in the reaching movement increased. However, when TMS was delivered at the time of movement onset, as triggered by muscle activity, cortical excitability decreased. Interestingly, motor performance significantly improved following training but did not differ between conditions. Thus, there is a very narrow time window available between cortical and peripheral activity to induce LTPlike and LTD-like plasticity.

\section{Homeostatic Metaplasticity}

Synaptic plasticity regulated by LTP and LTD can become excessive through activation of a positive feedback loop (Abraham, 2008; Murphy and Corbett, 2009). To counteract this positive feedback loop and maintain a physiological range of synaptic plasticity, negative-feedback is necessary (Murphy and Corbett, 2009). Homeostatic metaplasticity is a form of synaptic plasticity that modifies and maintains the stability of neuron and neuronal network activity within the physiological range (Abraham, 2008; Turrigiano, 2008; Murphy and Corbett, 2009). A conceptual basis of homeostatic metaplasticity is provided by the Bienenstock-Cooper-Munro (BCM) theory: the threshold for induction of LTP or LTD is dynamically adjusted according to the history of activation (Bienenstock et al., 1982). An increase in the synaptic modification threshold by recent high-level activity will favor the induction of LTD over LTP. Conversely, a reduction in the threshold due to low-level activity will favor the induction of LTP over LTD (Bienenstock et al., 1982).

In the human motor system, many studies have reported interactions between interventions via homeostatic metaplasticity by combining two non-invasive brain stimulation (NIBS) techniques, which can change cortical excitability (Iyer et al., 2003; Lang et al., 2004; Hamada et al., 2008; Fricke et al., 2011; Murakami et al., 2012). However, it is important for the continuous improvement of motor function after stroke that the combination of techniques for neurorehabilitation are based on motor learning, rather than NIBS intervention alone. Therefore, in this section, we mainly discuss homeostatic plasticity elicited when combining NIBS with motor learning.

Jung and Ziemann evaluated the correlation between LTP/LTD-like plasticity induced by PAS and motor learning in healthy subjects (Jung and Ziemann, 2009). They reported that this combination of facilitation techniques is influenced by homeostatic metaplasticity if there is an extended period of time between interventions. Motor training immediately following LTD-like plasticity enhanced motor learning according to homeostatic interactions. In addition, motor training immediately following LTP-like plasticity also enhanced motor learning, although to a lesser extent. However, if motor training was undertaken 90 min after PAS, LTD-like plasticity facilitated motor learning, whereas LTP-like plasticity depressed motor learning. Therefore, subsequent facilitation techniques occurring with a long time delay after the first facilitation program are easily influenced by homeostatic interactions, whereas a synergic effect of combined facilitatory approaches without a delay is expected because homeostatic interactions are avoided. Animal studies showing that non-saturated LTP facilitated subsequent learning may provide some explanation for the non-homeostatic interactions between LTP-like plasticity and immediately subsequent motor learning (Berger, 1984; Jeffery and Morris, 1993). These results indicate that neurorehabilitation based on motor learning immediately followed by a facilitation technique might avoid a reduction in the synergic effect due to homeostatic metaplasticity. This is consistent with another study showing that priming with excitatory intermittent theta burst stimulation (iTBS), with an interval of $10 \mathrm{~min}$ between iTBS and motor training, enhanced the subsequent motor learning of ballistic thumb movements (Teo et al., 2011). However, the effect of subsequent motor training may depend on the type of NIBS; Kuo et al. reported that excitatory anodal transcranial direct current stimulation (tDCS) over M1 immediately before a serial reaction time task does not affect implicit motor learning (Kuo et al., 2008). Conversely, Nitsche et al. demonstrated that the application of anodal tDCS during the same task leads to an improvement in implicit motor learning (Nitsche et al., 2003). Stagg et al. have also shown that anodal tDCS improves explicit motor learning when applied during the motor task, but not if it is applied before the task (Stagg et al., 2011). Therefore, homeostatic effects may occur in M1 when excitatory tDCS is applied before motor training that increases excitability in an activity-dependent manner.

On the other hand, simultaneous timing between interventions that are based on similar mechanisms is always not desirable for a synergic effect. Nitsche et al. investigated whether the timing between tDCS and LTP-like plasticity induced by PAS influences homeostatic metaplasticity (Nitsche et al., 2007). They reported that preconditioning M1 with excitatory tDCS enhances the subsequent PAS-induced LTP-like plasticity, whereas inhibitory tDCS reduces subsequent LTP-like plasticity. In contrast, when tDCS and LTP-like plasticity are applied simultaneously, inhibitory tDCS results in a prolonged LTP-like plasticity, whereas excitatory tDCS produces inhibition of LTPlike plasticity. Thus, the effects of homeostatic metaplasticity may differ depending on the type of combined interventions, in addition to their timing.

In next section, we discuss the combination therapies currently used to treat patients with stroke in the context of homeostatic metaplasticity and Hebbian plasticity. However, research findings describing homeostatic metaplasticity and Hebbian plasticity are mainly obtained from young healthy subjects. Moreover, there is considerable evidence that activitydependent plasticity after motor learning is reduced in older subjects compared to young ones (Sawaki et al., 2003; Zimerman et al., 2013). Furthermore, the neural plasticity induced by NIBS is different between young and older subjects (Ridding and Ziemann, 2010; Zimerman et al., 2013). Age-related neural plasticity changes are known to result from region-specific changes in dendritic morphology, cellular connectivity, $\mathrm{Ca}^{2+}$ dysregulation, and gene expression, amongst other factors (Burke and Barnes, 2006). In addition to aging, stroke itself induces structural and functional changes in the brain (Murphy and Corbett, 2009; Starkey and Schwab, 2014). Therefore, the effect of combinatorial interventions in patients with stroke might differ 
from those predicted from findings in young healthy subjects due to structural and functional changes in aging and/or neural reorganization after stroke (Burke and Barnes, 2006; Murphy and Corbett, 2009; Heise et al., 2013; Starkey and Schwab, 2014).

\section{Combined Neurorehabilititation Therapies for Motor Recovery after Stroke}

Over the last several decades, many studies have reported various motor learning-based stroke rehabilitation strategies (Langhorne et al., 2009; Johansson, 2012; Pollock et al., 2014; Veerbeek et al., 2014). Constraint-induced movement therapy (CIMT), which combines a rehabilitative training regime for the paretic limb with non-paretic limb restraint, can overcome learned nonuse of the paretic limb and has been shown to improve motor function in patients with stroke. Robotic training can provide repetitive motor training and intensive practice. NIBS and neuromuscular electrical stimulation can improve motor recovery by ameliorating use-dependent plasticity impairment after stroke. Moreover, stroke rehabilitation strategies such as mental practice, virtual reality, and mirror therapy have been developed based on multisensory feedback, which plays an important role in reestablishing the disrupted sensorimotor loop after stroke. The efficacy of each motor neurorehabilitation technique has been validated, however the additive effects of combined neurorehabilitation therapies remain to be elucidated. As described above, when different stroke neurorehabilitation therapies are combined, the timing of these programs must be considered in the context of Hebbian plasticity and homeostatic metaplasticity. In this section, we discuss these combined neurorehabilitation therapies in two sections describing different and simultaneous timing strategies, respectively.

\section{Different Timing between Neurorehabilitation Strategies}

The concept of priming has helped us to understand the interaction between different neurorehabilitation processes when interventions are performed separately. Behavioral, environmental, pharmacological, or electrophysiological stimulation have been utilized as priming strategies that modify the effects of a subsequent intervention. In particular, many studies have utilized NIBS, such as repetitive TMS (rTMS) and tDCS, to prime cortical stimulation because there is strong evidence that this can change cortical excitability (Pascual-Leone et al., 1994; Chen et al., 1997; Nitsche and Paulus, 2000 and for review, see Lefaucheur, 2009). At present, increasing the excitability of the ipsilesional M1 by NIBS has been extensively studied to enhance use-dependent plasticity induced by physical therapy (Hummel et al., 2005; Kim et al., 2006; Takeuchi et al., 2008; Di Lazzaro et al., 2010). The interhemispheric competition model after stroke suggests that the excitability of the ipsilesional M1 can be increased by direct excitatory NIBS over ipsilesional M1 or indirect inhibitory NIBS over the contralesional M1 via a reduction in excessive interhemispheric inhibition from the contralesional to ipsilesional M1 (Hummel et al., 2005; Takeuchi et al., 2005; Kim et al., 2006 and for reviews, see Hummel and Cohen, 2006; Takeuchi and Izumi, 2012b).
A previous study has shown the importance of using priming NIBS for motor training by administering either inhibitory $1 \mathrm{~Hz}$ or sham rTMS over the contralesional M1 immediately before or after physical therapy (Avenanti et al., 2012). rTMS improved motor function in the paretic hand, regardless of whether it was administered before or after physical therapy; however, the group receiving rTMS before physical therapy showed robust and stable improvements when compared with the group receiving rTMS after physical therapy. In addition, bilateral priming stimulation improves the effect of physical therapy in stroke patients. Takeuchi et al. investigated whether motor training after bilateral rTMS improves motor learning in the paretic hand of patients with chronic stroke (Takeuchi et al., 2009). They found that bilateral rTMS, which consisted of alternating 1 and $10 \mathrm{~Hz}$ rTMS over the contralesional and ipsilesional M1, respectively, improved the effect of subsequent motor training more than unilateral stimulation. Sung et al. have shown that a combined protocol of inhibitory rTMS over the contralesional M1 followed by excitatory iTBS over the ipsilesional M1 enhances the effect of the conventional physical rehabilitation when compared with that of unilateral stimulation alone (Sung et al., 2013).

However, it is noted that bilateral stimulation may reduce synergic effects due to homeostatic metaplasticity. Ragert et al. reported that bilateral stimulation obeys homeostatic mechanisms operating across hemispheric boundaries and regulates the excitability of $\mathrm{M} 1$ in a manner similar to that seen for unilateral stimulation in healthy subjects (Ragert et al., 2009). They showed that the application of either inhibitory $1 \mathrm{~Hz}$ rTMS over the right M1 or excitatory iTBS over the left M1 results in increased cortical excitability in the left M1 relative to sham interventions. Preconditioning with $1 \mathrm{~Hz}$ rTMS over the right M1 significantly attenuated the excitability-enhancing effect of subsequent iTBS over the left M1, in line with homeostatic metaplasticity. Therefore, the positive results indicating that bilateral stimulation in stroke patients had a synergic effect on motor recovery seem to operate through different mechanisms than conventional homeostatic metaplasticity in healthy subjects. A recent study that the order of bilateral hemisphere stimulation was important for synergic effects might suggest that bilateral stimulation in stroke patients is less influenced by homeostatic metaplasticity. Wang et al. performed either a priming protocol with inhibitory $1 \mathrm{~Hz}$ rTMS to the contralesional M1 and subsequent excitatory iTBS to the ipsilesional M1 or the reverse, followed by conventional rehabilitation in stroke patients (Wang et al., 2014a). The first combination induced a better improvement in hand function than the second combination. As a possible mechanism, inhibitory rTMS over the contralesional M1 in patients with stroke could not fully enhance the excitability of the ipsilesional $\mathrm{M} 1$, in contrast to the findings for healthy subjects; therefore, homeostatic metaplasticity might not occur with bilateral stimulation.

In addition to physical therapy, priming NIBS facilitates the effect of other stroke motor neurorehabilitation methods, such as robot training, virtual reality, peripheral sensorimotor stimulation, and CIMT (Table 1). As another form of priming, many studies have investigated whether pharmacological intervention improves motor learning in stroke patients. 


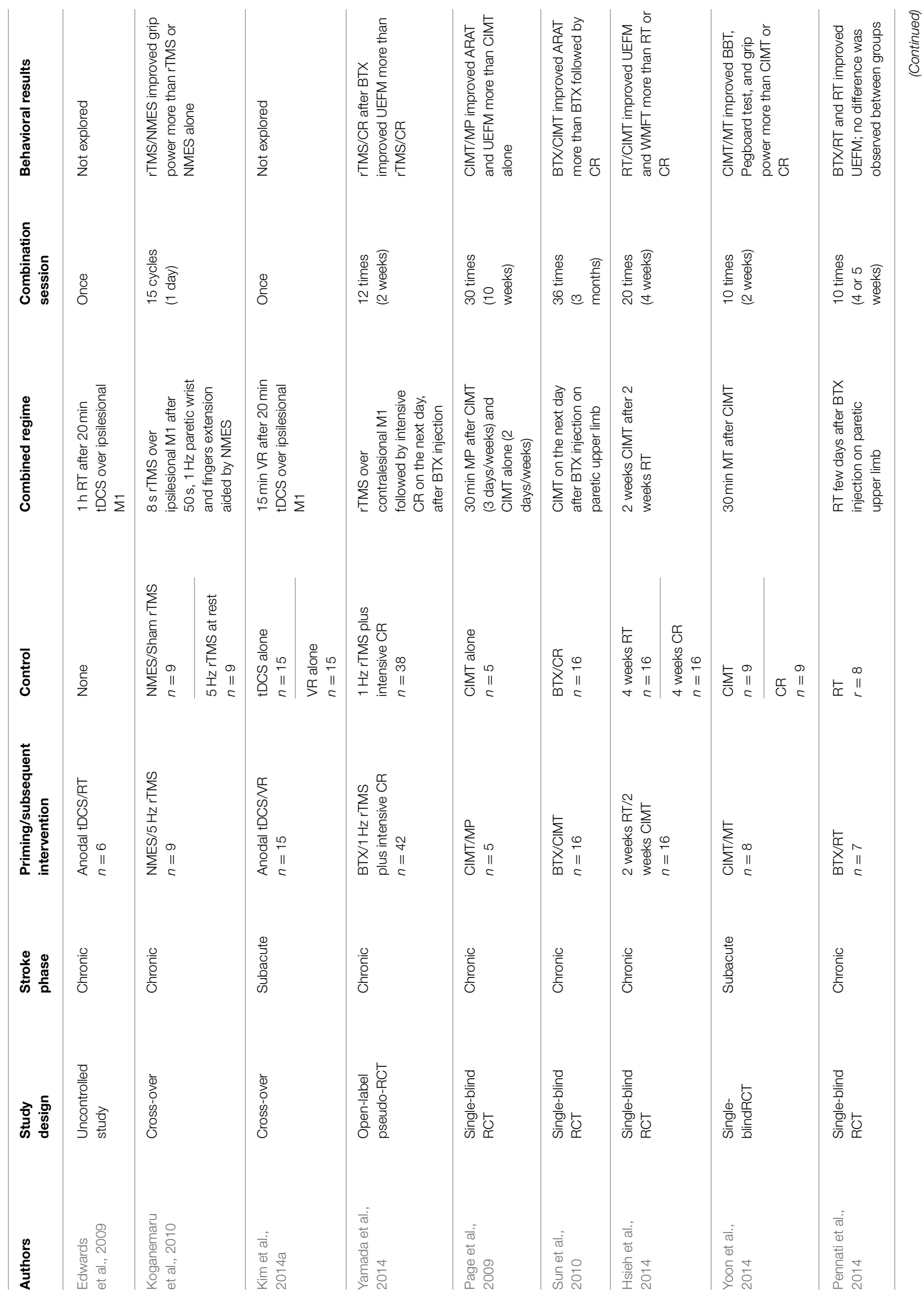




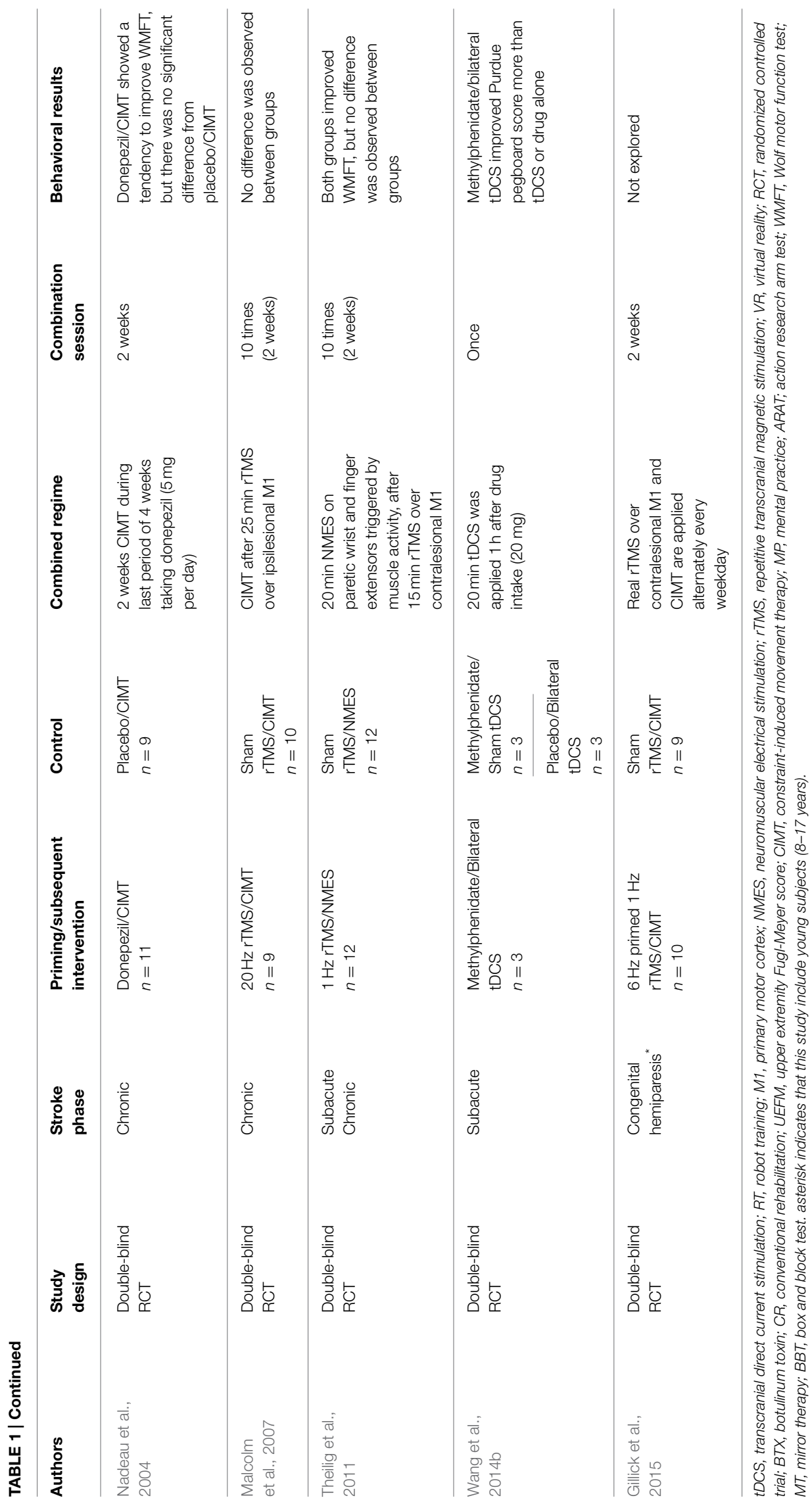


Clinical trials suggest that pharmacological interventions, such as dextroamphetamine (Schuster et al., 2011), selective serotonin reuptake inhibitors (Chollet et al., 2011), donepezil (Berthier et al., 2003), or levodopa (Rosser et al., 2008) may facilitate physical therapy. Pharmacological intervention is also expected to enhance the effect of stroke neurorehabilitation for motor recovery (Nadeau et al., 2004; Wang et al., 2014b). Furthermore, although this finding is slightly different from the concept of priming, several studies have reported that a combination of stroke neurorehabilitation therapies based on distinct mechanisms can improve motor function in stroke patients (Page et al., 2009; Sun et al., 2010; Hsieh et al., 2014; Yoon et al., 2014) (Table 1).

However, it should be noted that a few studies have reported no synergic effect in combining priming intervention with stroke neurorehabilitation (Malcolm et al., 2007; Theilig et al., 2011; Pennati et al., 2014). Moreover, it remains to be evaluated whether combined neurorehabilitation provides the most optimal approach for motor recovery. As described above, combining neurorehabilitation therapies that target different mechanisms and have shorter intervals might be desirable for avoiding homeostatic metaplasticity and creating a synergic effect. Future study must clarify these questions.

\section{Simultaneous Timing between Neurorehabilitation Therapies}

Unlike combined neurorehabilitation strategies employing different timing, simultaneous interventions may provide more closed-loop strategies that target Hebbian plasticity. In this section, we discuss simultaneous timing between neurorehabilitation therapies, assessing muscle activitydependent stimulation, brain state-dependent stimulation, and simultaneous combination neurorehabilitation therapies.

\section{Muscle Activity-Dependent Stimulation}

Several studies have reported electromyography (EMG)triggered neuromuscular electrical stimulation to induce motor recovery after stroke as combined sensorimotor stimulation synchronizes with muscle activity (Meilink et al., 2008; Shin et al., 2008; Fujiwara et al., 2009; Theilig et al., 2011). In addition, brain stimulation applied concurrently with voluntary movements is a form of muscle activity-dependent stimulation. Izumi et al. reported that muscle activity-dependent cortical stimulation synchronizes with hand movements in chronic stroke patients (Izumi et al., 2008). Nine patients were assigned to receive 100 pulses of active or sham $0.1 \mathrm{~Hz}$ TMS to the ipsilesional M1 during maximal effort paretic thumb and finger extension. Active TMS synchronized with maximum effort to induce a target movement that improved the motor function of the paretic hand when compared with sham TMS. Buetefisch et al. studied activitydependent TMS combined with robot-assisted motor training in six chronic stroke patients (Buetefisch et al., 2011). Patients executed robot-assisted wrist extension movements at $0.2 \mathrm{~Hz}$ frequency while subthreshold $0.1 \mathrm{~Hz}$ TMS triggered by muscle activity was applied to the ipsilesional M1. This stimulation produced different map reorganization representing the muscle in the training movement when compared with robot-assisted motor training alone. However, this study did not show any difference in motor function between TMS synchronized with robot training and robot training alone. Massie et al. investigated muscle activity-dependent cortical stimulation synchronized with paretic hand movement using rTMS in patients with chronic stroke (Massie et al., 2013). Eighteen patients were assigned to receive $10 \mathrm{~Hz}$ rTMS during voluntary movement or $10 \mathrm{~Hz}$ rTMS at rest. Patients in the rTMS during movement group had $30 \times 3 \mathrm{~s} 10 \mathrm{~Hz}$ rTMS over the ipsilesional M1 synchronized with lateral pinch contraction of the paretic hand. Activity-dependent rTMS with movement increased the cortical excitability of the ipsilesional M1 when compared with rTMS delivered during rest. However, there was no difference in pinch force between the two groups. At present, there is little evidence of muscle activity-dependent cortical stimulation in stroke patients. Moreover, the strict timing for LTP-like plasticity should be considered because brain stimulation that is delayed from movement onset reduces the excitability of the motor cortex in healthy subjects (Thabit et al., 2010; Massie et al., 2015).

\section{Brain State-Dependent Stimulation}

Muscle activity-dependent stimulation is not applicable for the stroke patients with severe hemiparesis who have no voluntary muscle activation. Therefore, these patients require activitydependent stimulation paradigms based on brain activity. This concept is called brain state-dependent stimulation and has been developed in mainly animal studies using the action potentials of single neurons from an implanted electrode (Jackson et al., 2006; Rebesco et al., 2010). Recently, brain state-dependent stimulation has been investigated in humans via the development of braincomputer interface (BCI) techniques that can monitor brain activity in real time. Brain state-dependent stimulation using a BCI typically utilizes robot-assisted movement execution that is synchronized with brain activity during voluntary movement intention. It is postulated that the re-establishment of the disrupted sensorimotor loop by integrating movement intention and passive limb movement, assisted by a robot, will strengthen the associative connection, following the principles of Hebbian plasticity. Ramos et al. investigated whether robotic orthosis feedback using an electroencephalograph (EEG)-based BCI could improve physical therapy (Ramos-Murguialday et al., 2013). Thirty chronic stroke patients with severe hand weakness were randomly assigned to either the relevant or irrelevant feedback group. The results showed that the relevant online orthosis feedback improved subsequent physical therapy in chronic stroke patients with severe hemiparesis when compared with the random irrelevant feedback group.

Ang et al. has investigated whether an EEG-based motor imagery (MI) BCI coupled with robot-assisted hand grasping has an additional effect on therapist-assisted arm mobilization (Ang et al., 2014b). Twenty-one patients with chronic stroke were randomly allocated to the MI BCI coupled with robot feedback (BCI-robot training), robot training, or standard arm therapy group. Eighteen sessions of BCI-robot training led to additional improvement in therapist-assisted arm mobilization when compared with standard arm therapy; however, there was no significant difference between BCI-robot training and 
robot training alone. Another study by this group reported that sensorimotor feedback using a BCI had no additional effect on robot training-based neurorehabilitation (Ang et al., 2014a). Thus, using brain state-dependent activity stimulation as a neurorehabilitation therapy is very attractive, but still in its infancy; therefore, the optimal timing between brain activity and haptic feedback for Hebbian plasticity is yet to be elucidated.

\section{Simultaneous Combination Neurorehabilitation Therapies}

tDCS has an advantage in that it can be simultaneously performed with other interventions, as it is not limited by movement. Therefore, many researchers have explored simultaneous tDCS and physical therapy to improve motor function in stroke patients (Lindenberg et al., 2012; Stagg et al., 2012; Zimerman et al., 2012). As with priming strategies, it has been reported that bilateral stimulation using tDCS during physical therapy also improved motor function in patients with stroke (Lindenberg et al., 2010b, 2012). tDCS during motor training might avoid a reduction in synergic effects due to homeostatic metaplasticity in patients with stroke as well as healthy subjects (Nitsche et al., 2003; Stagg et al., 2011). However, it remains to be evaluated whether tDCS before or during motor training is optimal for motor recovery, because at the present there is no evidence that simultaneous tDCS and motor training is more effective for motor recovery than priming tDCS. Moreover, to our knowledge, there are no reports describing activity-dependent tDCS in which short duration tDCS is repeatedly paired with volitional activity in order to induce Hebbian plasticity in a strict temporal relationship.

In addition to physical therapy, several studies have reported that the use of tDCS during neurorehabilitation procedures such as peripheral nerve stimulation, robot-assisted arm training, virtual reality training, and CIMT, facilitates motor recovery after stroke (Celnik et al., 2009; Bolognini et al., 2011; Ochi et al., 2013; Lee and Chun, 2014) (Table 2). As with different timing strategies, other simultaneous combined stroke neurorehabilitation therapies have been investigated to improve motor recovery (Reinkensmeyer et al., 2012; Mihara et al., 2013; Kim and Lee, 2015; Lin et al., 2014) (Table 2). Considering concepts of simultaneous timing, multisensory stimulation strategies such as motor imagery, mirror therapy, and virtual reality might be easy to combine with neurorehabilitation based on motor training. However, it remains to be evaluated which combined neurorehabilitation approaches are effective for motor recovery. Moreover, simultaneous combinations of neurorehabilitation approaches are not always synergistically effective for motor recovery in stroke patients. Hesse et al. studied the use of simultaneous tDCS and robot-assisted arm training in subacute stroke patients with severe motor function deficits and found that neither inhibitory tDCS over the contralesional M1 nor excitatory tDCS over the ipsilesional M1 enhanced the effect of robot-assisted arm training when compared with robot-assisted training alone (Hesse et al., 2011). Although, this study included patients with severe motor deficit, it should be considered that homeostatic plasticity might reduce synergic effects even in the case of simultaneous combinations.
In line with this, Nitsche et al. suggested that simultaneous excitatory tDCS and LTP-like plasticity induced by PAS reduces corticospinal excitability in healthy subjects (Nitsche et al., 2007).

Thus, many studies have reported positive synergic effects of combined neurorehabilitation therapies in both different and simultaneous timing strategies; however, the outcome of these combination-based strategies is influenced by several factors. At the present, there have been many reports of the use of neurorehabilitation combinations that include NIBS. NIBS combination strategies use the interhemispheric competition model after stroke as rationale; however, applying this theory to all patients with stroke is oversimplified or even incorrect (Di Pino et al., 2014). In fact, several studies have reported that NIBS might have no effect of motor recovery in some stroke patients with acute phase or cortical lesions (Ameli et al., 2009; Seniow et al., 2012; Rossi et al., 2013). Moreover, in contrast to the usual NIBS strategies based on the interhemispheric competition model, an exploratory study has reported that priming with an inhibitory continuous TBS (cTBS) over the ipsilesional M1 improved subsequent motor training in the paretic upper extremity through homeostatic metaplasticity (Di Lazzaro et al., 2013). These results indicate that the effect of combination therapies depends not only on the timing, type and intensity of intervention, but also phase and lesion site after stroke, possibly due to heterogeneous neural reorganization responses to stroke. Similarly, homeostatic metaplasticity and Hebbian plasticity might vary according to stroke state. In future research, neurophysiological and functional neuroimaging studies must elucidate the mechanisms of heterogeneous responses to combined therapies in patients with stroke. In addition to clinical measurement, such as severity of motor function, the evaluation of corticospinal tract integrity and lesion size/location using neurophysiological and neuroimaging techniques also may be useful to control for variability of stroke lesions (Stinear et al., 2007; Lindenberg et al., 2010a; Zhu et al., 2010). In next section, we discuss methods for facilitating combination therapy in the context of homeostatic metaplasticity and Hebbian plasticity.

\section{Facilitating the Synergic Effect of Combined Neurorehabilitation Therapies}

The synergic effect of combined neurorehabilitation therapies will vary according to the type and timing of the neurorehabilitation used. To facilitate this synergic effect, it is important to predict its effect by confirming the mechanism involved, in addition to improving new technologies. We have mainly discussed peripheral sensorimotor feedback as a brain-state dependent stimulation, but simultaneous cortical and peripheral stimulation using BCI technologies could provide a novel neurorehabilitation strategy for stroke patients. Preliminary work decoding brain signals of motor imagery has shown that synchronous TMS with haptic feedback assisted by a robot facilitated the induction of associative plasticity (Gharabaghi et al., 2014). In addition to synchronous cortical and peripheral stimulation, real-time monitoring of 


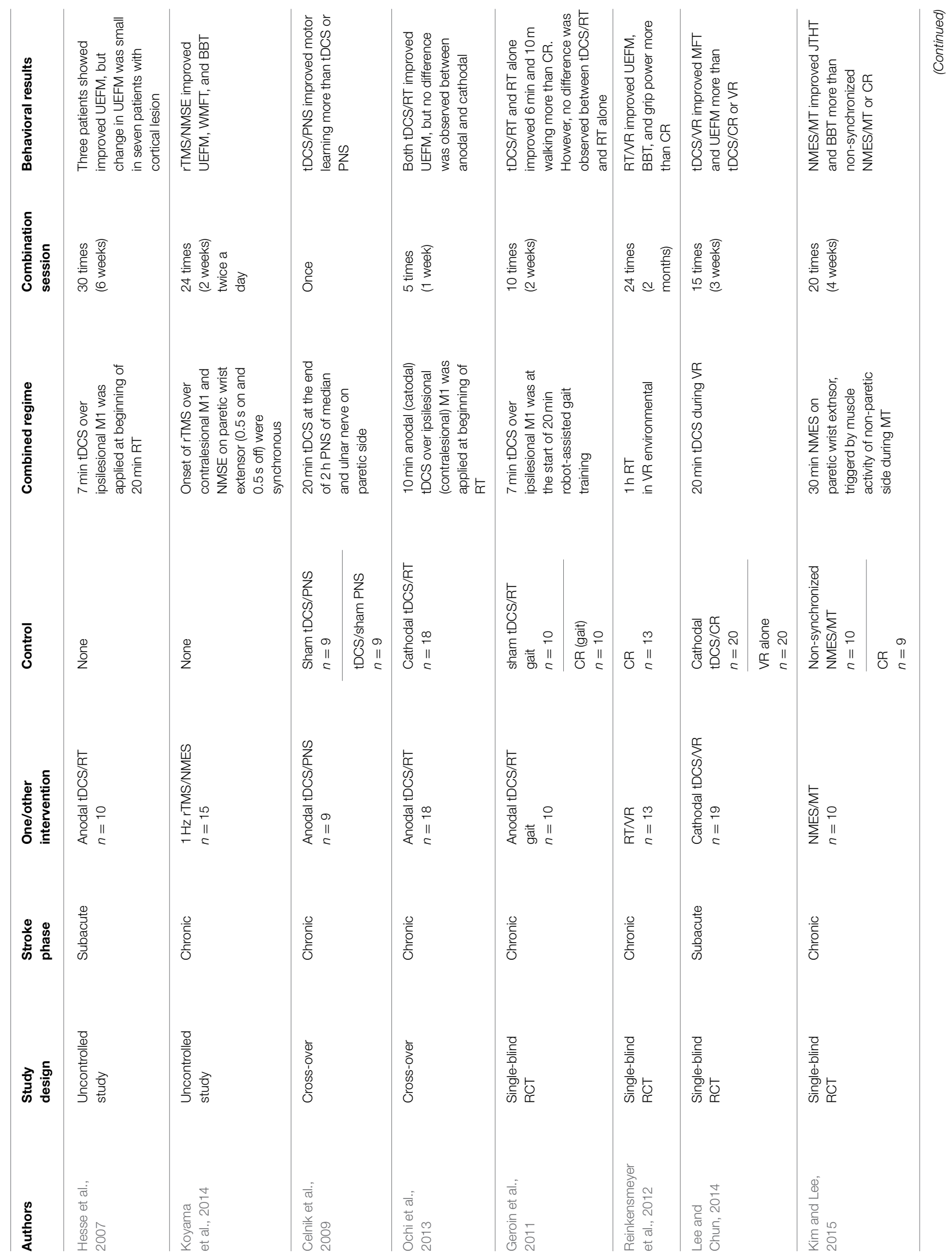




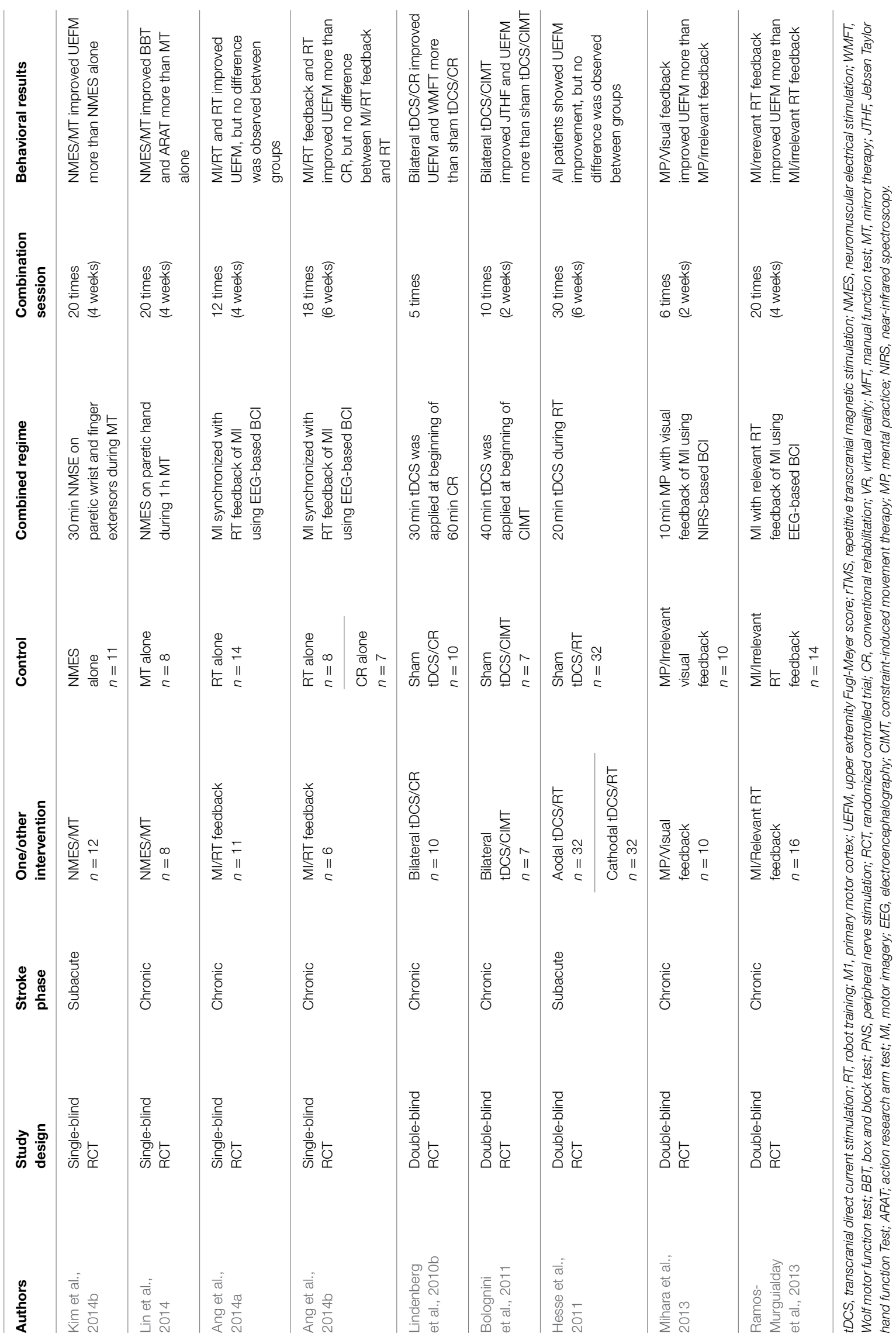


brain activity will reveal the optimal timing between different neurorehabilitation therapies. The development of technology that decodes brain activity might facilitate the synergic effect of combined stroke neurorehabilitation by monitoring the intrinsic variations in neuronal excitability, which might influence homeostatic metaplasticity and Hebbian plasticity.

The synergic effect might be facilitated when it is combined with multisensory simulation, such as motor imagery, action observation, mental practice, mirror therapy, training in virtual reality, and music-related therapies (Johansson, 2012). Multisensory training protocols better approximate natural settings and are more effective for learning (Shams and Seitz, 2008; Johansson, 2011). Moreover, multisensory stimulation strategies induce activation of higher order association areas as well as unimodal sensory areas (Ghazanfar and Schroeder, 2006; Johansson, 2012). Therefore, multiple regions activated by multisensory stimulation might have associative connections with the motor cortex, resulting in enhanced Hebbian plasticity. Moreover, combination neurorehabilitation strategies based on motor learning and multisensory stimulation might avoid a convergence of LTP-like plasticity on M1 that reduces the synergic effect via homeostatic metaplasticity. Furthermore, the effects of emotional motivation that can be activated by multisensory stimulation, such as music and virtual reality interactive games, could engage reward-learning networks, helping to facilitate the motor recovery (Camara et al., 2009; Rodriguez-Fornells et al., 2012; Novak et al., 2014).

If associative plasticity between multiple regions and M1 is important for the synergic effect of combined neurorehabilitation, direct brain stimulation outside M1 may also enhance the neurorehabilitation. Although we have primarily discussed $\mathrm{M} 1$ as the brain stimulation site for neurorehabilitation, several studies have shown neuroplastic changes induced by stimulating other areas of the brain that are functionally connected to M1, such as the somatosensory (Bliem et al., 2008), supplementary (Hamada et al., 2009), and premotor (Potter-Nerger et al., 2009) cortices, and the cerebellum (Popa et al., 2013). A previous study has revealed that inhibitory cTBS to both the contralesional M1 and primary somatosensory cortex can enhance motor improvement owed to practice with the paretic hand in patients with chronic stroke when compared with sham stimulation (Meehan et al., 2011). Moreover, the parameters of improvements in motor function were different between stimulation over M1 and the somatosensory cortex, indicating that stimulation beyond M1 may have a differential impact on stroke rehabilitation. Activation of movement-related components such as sensation, proprioception, motor planning, and coordination might enhance motor recovery by new associative plasticity pathways beyond M1.

In this regard, functional and structural connectivity neuroimaging information will be helpful in better understanding the heterogeneity of associative plasticity and to allow the individual design of combined neurorehabilitation programs based on preserved and undamaged brain connectivity (Rehme et al., 2011; Grefkes and Ward, 2014). Moreover, the response to NIBS might reveal how homeostatic plasticity influences the synergic effect of combined neurorehabilitation therapies in patients with stroke. Several studies using NIBS have provided evidence regarding homeostatic metaplasticity (Lang et al., 2004; Fricke et al., 2011; Murakami et al., 2012). Many studies have assessed the combination of facilitatory techniques in patients with stroke; however, these approaches risk degradation of subsequent learning according to homeostatic metaplasticity. The threshold and degree of homeostatic metaplasticity itself might change after stroke, which may contribute to the mixed results seen with combined neurorehabilitation therapies. Therefore, NIBS programs could serve as diagnostic tools of responsiveness to combined neurorehabilitation therapies by evaluating the threshold and degree of homeostatic metaplasticity in individual patients with stroke.

Plasticity outside the brain is of interest in future studies using combined neurorehabilitation, as plasticity may occur in the spinal cord (Taylor and Martin, 2009). In particular, it is noted that the reorganization of spinal cord can influence the synergic effect of combined neurorehabilitation on gait disturbance (Geroin et al., 2011), because walking ability is related to both cortical and spinal levels of organization (central pattern generator). There is little evidence to date regarding plasticity in the spinal cord following neurorehabilitation (MottaOishi et al., 2013; Zheng et al., 2013); however, structural plasticity is known to occur after stroke in animal studies (for review, see Starkey and Schwab, 2014).

\section{Conclusion}

Although meta-analysis and systematic reviews have investigated these therapies, there is a lack of convincing evidence to support any motor stroke neurorehabilitation approach as being more effective in recovery than any other approach (Langhorne et al., 2009; Hsu et al., 2012; Pollock et al., 2014; Veerbeek et al., 2014). Moreover, the effectiveness of motor neurorehabilitation varies across patients with stroke. Therefore, these techniques are being investigated to determine whether their combination has stable and synergistic effects in motor recovery. Nevertheless, combination techniques have shown mixed results. There is heterogeneity in the combined neurorehabilitation protocols that are used, for example, with respect to factors such as the number of sessions, intensity, type and timing of interventions, time after stroke, lesion site, severity of motor function, gender, pathology, and genetic factors, which adds complexity to this field. The change in the threshold and degree of Hebbian plasticity and/or homeostatic metaplasticity after stroke might influence the uncertainty of the synergic effect of combined neurorehabilitation. Few possible combinations of stroke neurorehabilitation have been tested experimentally; therefore, utilizing the concept of Hebbian plasticity and homeostatic metaplasticity can help in developing an appropriate combination of therapies for motor recovery in future studies.

\section{Acknowledgments}

This work was supported by JSPS Grant-in-Aid for Scientific Research Number 25242054. 


\section{References}

Abraham, W. C. (2008). Metaplasticity: tuning synapses and networks for plasticity. Nat. Rev. Neurosci. 9, 387-399. doi: 10.1038/nrn2356

Ameli, M., Grefkes, C., Kemper, F., Riegg, F. P., Rehme, A. K., Karbe, H., et al. (2009). Differential effects of high-frequency repetitive transcranial magnetic stimulation over ipsilesional primary motor cortex in cortical and subcortical middle cerebral artery stroke. Ann. Neurol. 66, 298-309. doi: 10.1002/ana.21725

Ang, K. K., Chua, K. S., Phua, K. S., Wang, C., Chin, Z. Y., Kuah, C. W., et al. (2014a). A randomized controlled trial of EEG-based motor imagery braincomputer interface robotic rehabilitation for stroke. Clin. EEG Neurosci. doi: 10.1177/1550059414522229. [Epub ahead of print].

Ang, K. K., Guan, C., Phua, K. S., Wang, C., Zhou, L., Tang, K. Y., et al. (2014b). Brain-computer interface-based robotic end effector system for wrist and hand rehabilitation: results of a three-armed randomized controlled trial for chronic stroke. Front. Neuroeng. 7:30. doi: 10.3389/fneng.2014.00030

Avenanti, A., Coccia, M., Ladavas, E., Provinciali, L., and Ceravolo, M. G. (2012). Low-frequency rTMS promotes use-dependent motor plasticity in chronic stroke: a randomized trial. Neurology 78, 256-264. doi: 10.1212/WNL.0b013e3182436558

Berger, T. W. (1984). Long-term potentiation of hippocampal synaptic transmission affects rate of behavioral learning. Science 224, 627-630. doi: $10.1126 /$ science. 6324350

Berthier, M. L., Pujol, J., Gironell, A., Kulisevsky, J., Deus, J., Hinojosa, J., et al. (2003). Beneficial effect of donepezil on sensorimotor function after stroke. Am. J. Phys. Med. Rehabil. 82, 725-729. doi: 10.1097/01.PHM.0000083668.48396.84

Bienenstock, E. L., Cooper, L. N., and Munro, P. W. (1982). Theory for the development of neuron selectivity: orientation specificity and binocular interaction in visual cortex. J. Neurosci. 2, 32-48.

Bliem, B., Muller-Dahlhaus, J. F., Dinse, H. R., and Ziemann, U. (2008). Homeostatic metaplasticity in the human somatosensory cortex. J. Cogn. Neurosci. 20, 1517-1528. doi: 10.1162/jocn.2008.20106

Bolognini, N., Vallar, G., Casati, C., Latif, L. A., El-Nazer, R., Williams, J., et al. (2011). Neurophysiological and behavioral effects of tDCS combined with constraint-induced movement therapy in poststroke patients. Neurorehabil. Neural Repair 25, 819-829. doi: 10.1177/1545968311411056

Buetefisch, C., Heger, R., Schicks, W., Seitz, R., and Netz, J. (2011). Hebbian-type stimulation during robot-assisted training in patients with stroke. Neurorehabil. Neural Repair 25, 645-655. doi: 10.1177/1545968311402507

Burke, S. N., and Barnes, C. A. (2006). Neural plasticity in the ageing brain. Nat. Rev. Neurosci. 7, 30-40. doi: 10.1038/nrn1809

Butefisch, C. M., Davis, B. C., Wise, S. P., Sawaki, L., Kopylev, L., Classen, J., et al. (2000). Mechanisms of use-dependent plasticity in the human motor cortex. Proc. Natl. Acad. Sci. U.S.A. 97, 3661-3665. doi: 10.1073/pnas.97.7.3661

Butefisch, C. M., Khurana, V., Kopylev, L., and Cohen, L. G. (2004). Enhancing encoding of a motor memory in the primary motor cortex by cortical stimulation. J. Neurophysiol. 91, 2110-2116. doi: 10.1152/jn.01038.2003

Camara, E., Rodriguez-Fornells, A., Ye, Z., and Munte, T. F. (2009). Reward networks in the brain as captured by connectivity measures. Front. Neurosci. 3, 350-362. doi: 10.3389/neuro.01.034.2009

Celnik, P., Paik, N. J., Vandermeeren, Y., Dimyan, M., and Cohen, L. G. (2009). Effects of combined peripheral nerve stimulation and brain polarization on performance of a motor sequence task after chronic stroke. Stroke 40, 1764-1771. doi: 10.1161/STROKEAHA. 108.540500

Chen, R., Classen, J., Gerloff, C., Celnik, P., Wassermann, E. M., Hallett, M., et al. (1997). Depression of motor cortex excitability by low-frequency transcranial magnetic stimulation. Neurology 48, 1398-1403. doi: 10.1212/WNL.48.5.1398

Chollet, F., Tardy, J., Albucher, J. F., Thalamas, C., Berard, E., Lamy, C., et al. (2011). Fluoxetine for motor recovery after acute ischaemic stroke (FLAME): a randomised placebo-controlled trial. Lancet Neurol. 10, 123-130. doi: 10.1016/S1474-4422(10)70314-8

Classen, J., Liepert, J., Wise, S. P., Hallett, M., and Cohen, L. G. (1998). Rapid plasticity of human cortical movement representation induced by practice. J. Neurophysiol. 79, 1117-1123.

De Vico Fallani, F., Pichiorri, F., Morone, G., Molinari, M., Babiloni, F., Cincotti, F., et al. (2013). Multiscale topological properties of functional brain networks during motor imagery after stroke. Neuroimage 83, 438-449. doi: 10.1016/j.neuroimage.2013.06.039
Di Lazzaro, V., Profice, P., Pilato, F., Capone, F., Ranieri, F., Pasqualetti, P., et al. (2010). Motor cortex plasticity predicts recovery in acute stroke. Cereb. Cortex 20, 1523-1528. doi: 10.1093/cercor/bhp216

Di Lazzaro, V., Rothwell, J. C., Talelli, P., Capone, F., Ranieri, F., Wallace, A. C., et al. (2013). Inhibitory theta burst stimulation of affected hemisphere in chronic stroke: a proof of principle, sham-controlled study. Neurosci. Lett. 553, 148-152. doi: 10.1016/j.neulet.2013.08.013

Dimyan, M. A., and Cohen, L. G. (2011). Neuroplasticity in the context of motor rehabilitation after stroke. Nat. Rev. Neurol. 7, 76-85. doi: 10.1038/nrneurol.2010.200

Di Pino, G., Pellegrino, G., Assenza, G., Capone, F., Ferreri, F., Formica, D., et al. (2014). Modulation of brain plasticity in stroke: a novel model for neurorehabilitation. Nat. Rev. Neurol. 10, 597-608. doi: 10.1038/nrneurol.2014.162

Edwards, D. J., Krebs, H. I., Rykman, A., Zipse, J., Thickbroom, G. W., Mastaglia, F. L., et al. (2009). Raised corticomotor excitability of M1 forearm area following anodal tDCS is sustained during robotic wrist therapy in chronic stroke. Restor. Neurol. Neurosci. 27, 199-207. doi: 10.3233/RNN-2009-0470

Edwardson, M. A., Lucas, T. H., Carey, J. R., and Fetz, E. E. (2013). New modalities of brain stimulation for stroke rehabilitation. Exp. Brain Res. 224, 335-358. doi: 10.1007/s00221-012-3315-1

Fricke, K., Seeber, A. A., Thirugnanasambandam, N., Paulus, W., Nitsche, M. A., and Rothwell, J. C. (2011). Time course of the induction of homeostatic plasticity generated by repeated transcranial direct current stimulation of the human motor cortex. J. Neurophysiol. 105, 1141-1149. doi: 10.1152/jn.00608.2009

Fujiwara, T., Kasashima, Y., Honaga, K., Muraoka, Y., Tsuji, T., Osu, R., et al. (2009). Motor improvement and corticospinal modulation induced by hybrid assistive neuromuscular dynamic stimulation (HANDS) therapy in patients with chronic stroke. Neurorehabil. Neural Repair 23, 125-132. doi: $10.1177 / 1545968308321777$

Geroin, C., Picelli, A., Munari, D., Waldner, A., Tomelleri, C., and Smania, N. (2011). Combined transcranial direct current stimulation and robot-assisted gait training in patients with chronic stroke: a preliminary comparison. Clin. Rehabil. 25, 537-548. doi: 10.1177/0269215510389497

Gharabaghi, A., Kraus, D., Leao, M. T., Spuler, M., Walter, A., Bogdan, M., et al. (2014). Coupling brain-machine interfaces with cortical stimulation for brain-state dependent stimulation: enhancing motor cortex excitability for neurorehabilitation. Front. Hum. Neurosci. 8:122. doi: 10.3389/fnhum.2014.00122

Ghazanfar, A. A., and Schroeder, C. E. (2006). Is neocortex essentially multisensory? Trends. Cogn. Sci. 10, 278-285. doi: 10.1016/j.tics.2006.04.008

Gillick, B. T., Krach, L. E., Feyma, T., Rich, T. L., Moberg, K., Menk, J., et al. (2015). Safety of primed repetitive transcranial magnetic stimulation and modified constraint-induced movement therapy in a randomized controlled trial in pediatric hemiparesis. Arch. Phys. Med. Rehabil. 96, S104-S113. doi: 10.1016/j.apmr.2014.09.012

Grefkes, C., and Ward, N. S. (2014). Cortical reorganization after stroke: how much and how functional? Neuroscientist 20, 56-70. doi: 10.1177/1073858413491147

Hamada, M., Hanajima, R., Terao, Y., Okabe, S., Nakatani-Enomoto, S., Furubayashi, T., et al. (2009). Primary motor cortical metaplasticity induced by priming over the supplementary motor area. J. Physiol. 587, 4845-4862. doi: 10.1113/jphysiol.2009.179101

Hamada, M., Terao, Y., Hanajima, R., Shirota, Y., Nakatani-Enomoto, S., Furubayashi, T., et al. (2008). Bidirectional long-term motor cortical plasticity and metaplasticity induced by quadripulse transcranial magnetic stimulation. J. Physiol. 586, 3927-3947. doi: 10.1113/jphysiol.2008.152793

Hebb, D. O. (1949). The Organization of Behavior: A Neuropsychological Theory. New York, NY: Wiley.

Heise, K. F., Zimerman, M., Hoppe, J., Gerloff, C., Wegscheider, K., and Hummel, F. C. (2013). The aging motor system as a model for plastic changes of GABAmediated intracortical inhibition and their behavioral relevance. J. Neurosci. 33, 9039-9049. doi: 10.1523/JNEUROSCI.4094-12.2013

Hesse, S., Waldner, A., Mehrholz, J., Tomelleri, C., Pohl, M., and Werner, C. (2011). Combined transcranial direct current stimulation and robot-assisted arm training in subacute stroke patients: an exploratory, randomized multicenter trial. Neurorehabil. Neural Repair 25, 838-846. doi: 10.1177/154596831 1413906 
Hesse, S., Werner, C., Schonhardt, E. M., Bardeleben, A., Jenrich, W., and Kirker, S. G. (2007). Combined transcranial direct current stimulation and robot-assisted arm training in subacute stroke patients: a pilot study. Restor. Neurol. Neurosci. 25, 9-15.

Hsieh, Y. W., Lin, K. C., Horng, Y. S., Wu, C. Y., Wu, T. C., and Ku, F. L. (2014). Sequential combination of robot-assisted therapy and constraint-induced therapy in stroke rehabilitation: a randomized controlled trial. J. Neurol. 261, 1037-1045. doi: 10.1007/s00415-014-7345-4

Hsu, W. Y., Cheng, C. H., Liao, K. K., Lee, I. H., and Lin, Y. Y. (2012). Effects of repetitive transcranial magnetic stimulation on motor functions in patients with stroke: a meta-analysis. Stroke 43, 1849-1857. doi: 10.1161/STROKEAHA.111.649756

Hummel, F., Celnik, P., Giraux, P., Floel, A., Wu, W. H., Gerloff, C., et al. (2005). Effects of non-invasive cortical stimulation on skilled motor function in chronic stroke. Brain 128, 490-499. doi: 10.1093/brain/awh369

Hummel, F. C., and Cohen, L. G. (2006). Non-invasive brain stimulation: a new strategy to improve neurorehabilitation after stroke? Lancet Neurol. 5, 708-712. doi: 10.1016/S1474-4422(06)70525-7

Iyer, M. B., Schleper, N., and Wassermann, E. M. (2003). Priming stimulation enhances the depressant effect of low-frequency repetitive transcranial magnetic stimulation. J. Neurosci. 23, 10867-10872.

Izumi, S., Kondo, T., and Shindo, K. (2008). Transcranial magnetic stimulation synchronized with maximal movement effort of the hemiplegic hand after stroke: a double-blinded controlled pilot study. J. Rehabil. Med. 40, 49-54. doi: 10.2340/16501977-0133

Jackson, A., Mavoori, J., and Fetz, E. E. (2006). Long-term motor cortex plasticity induced by an electronic neural implant. Nature 444, 56-60. doi: 10.1038 /nature 05226

Jeffery, K. J., and Morris, R. G. (1993). Cumulative long-term potentiation in the rat dentate gyrus correlates with, but does not modify, performance in the water maze. Hippocampus 3, 133-140. doi: 10.1002/hipo.450030205

Johansson, B. B. (2011). Current trends in stroke rehabilitation. A review with focus on brain plasticity. Acta Neurol. Scand. 123, 147-159. doi: 10.1111/j.16000404.2010.01417.x

Johansson, B. B. (2012). Multisensory stimulation in stroke rehabilitation. Front. Hum. Neurosci. 6:60. doi: 10.3389/fnhum.2012.00060

Jung, P., and Ziemann, U. (2009). Homeostatic and nonhomeostatic modulation of learning in human motor cortex. J. Neurosci. 29, 5597-5604. doi: 10.1523/JNEUROSCI.0222-09.2009

Kim, H., Lee, G., and Song, C. (2014b). Effect of functional electrical stimulation with mirror therapy on upper extremity motor function in poststroke patients. J. Stroke Cerebrovasc. Dis. 23, 655-661. doi: 10.1016/j.jstrokecerebrovasdis.2013.06.017

Kim, J. H., and Lee, B. H. (2015). Mirror therapy combined with biofeedback functional electrical stimulation for motor recovery of upper extremities after stroke: a pilot randomized controlled Trial. Occup. Ther. Int. 22, 51-60. doi: 10.1002/oti.1384

Kim, Y. H., You, S. H., Ko, M. H., Park, J. W., Lee, K. H., Jang, S. H., et al. (2006). Repetitive transcranial magnetic stimulation-induced corticomotor excitability and associated motor skill acquisition in chronic stroke. Stroke 37, 1471-1476. doi: 10.1161/01.STR.0000221233.55497.51

Kim, Y. J., Ku, J., Cho, S., Kim, H. J., Cho, Y. K., Lim, T., et al. (2014a). Facilitation of corticospinal excitability by virtual reality exercise following anodal transcranial direct current stimulation in healthy volunteers and subacute stroke subjects. J. Neuroeng. Rehabil. 11:124. doi: 10.1186/1743-000311-124

Koganemaru, S., Mima, T., Thabit, M. N., Ikkaku, T., Shimada, K., Kanematsu, M., et al. (2010). Recovery of upper-limb function due to enhanced usedependent plasticity in chronic stroke patients. Brain 133, 3373-3384. doi: 10.1093/brain/awq193

Koyama, S., Tanabe, S., Warashina, H., Kaneko, T., Sakurai, H., Kanada, Y., et al. (2014). NMES with rTMS for moderate to severe dysfunction after stroke. NeuroRehabilitation 35, 363-368. doi: 10.3233/NRE-141127

Kuo, M. F., Unger, M., Liebetanz, D., Lang, N., Tergau, F., Paulus, W., et al. (2008). Limited impact of homeostatic plasticity on motor learning in humans. Neuropsychologia 46, 2122-2128. doi: 10.1016/j.neuropsychologia.2008.02.023

Lang, N., Siebner, H. R., Ernst, D., Nitsche, M. A., Paulus, W., Lemon, R. N., et al. (2004). Preconditioning with transcranial direct current stimulation sensitizes the motor cortex to rapid-rate transcranial magnetic stimulation and controls the direction of after-effects. Biol. Psychiatry 56, 634-639. doi: 10.1016/j.biopsych.2004.07.017

Langhorne, P., Bernhardt, J., and Kwakkel, G. (2011). Stroke rehabilitation. Lancet 377, 1693-1702. doi: 10.1016/S0140-6736(11)60325-5

Langhorne, P., Coupar, F., and Pollock, A. (2009). Motor recovery after stroke: a systematic review. Lancet Neurol. 8, 741-754. doi: 10.1016/S14744422(09)70150-4

Lee, S. J., and Chun, M. H. (2014). Combination transcranial direct current stimulation and virtual reality therapy for upper extremity training in patients with subacute stroke. Arch. Phys. Med. Rehabil. 95, 431-438. doi: 10.1016/j.apmr.2013.10.027

Lefaucheur, J. P. (2009). Methods of therapeutic cortical stimulation. Neurophysiol. Clin. 39, 1-14. doi: 10.1016/j.neucli.2008.11.001

Lin, K. C., Chen, Y. T., Huang, P. C., Wu, C. Y., Huang, W. L., Yang, H. W., et al. (2014). Effect of mirror therapy combined with somatosensory stimulation on motor recovery and daily function in stroke patients: a pilot study. J. Formos. Med. Assoc. 113, 422-428. doi: 10.1016/j.jfma.2012.08.008

Lindenberg, R., Renga, V., Zhu, L. L., Betzler, F., Alsop, D., and Schlaug, G. (2010a) Structural integrity of corticospinal motor fibers predicts motor impairment in chronic stroke. Neurology 74, 280-287. doi: 10.1212/WNL.0b013e3181ccc6d9

Lindenberg, R., Renga, V., Zhu, L. L., Nair, D., and Schlaug, G. (2010b). Bihemispheric brain stimulation facilitates motor recovery in chronic stroke patients. Neurology 75, 2176-2184. doi: 10.1212/WNL.0b013e318202013a

Lindenberg, R., Zhu, L. L., and Schlaug, G. (2012). Combined central and peripheral stimulation to facilitate motor recovery after stroke: the effect of number of sessions on outcome. Neurorehabil. Neural Repair 26, 479-483. doi: $10.1177 / 1545968311427568$

Liuzzi, G., Horniss, V., Lechner, P., Hoppe, J., Heise, K., Zimerman, M., et al. (2014). Development of movement-related intracortical inhibition in acute to chronic subcortical stroke. Neurology 82, 198-205. doi: 10.1212/WNL.0000000000000028

Malcolm, M. P., Triggs, W. J., Light, K. E., Gonzalez Rothi, L. J., Wu, S., Reid, K., et al. (2007). Repetitive transcranial magnetic stimulation as an adjunct to constraint-induced therapy: an exploratory randomized controlled trial. Am. J. Phys. Med. Rehabil. 86, 707-715. doi: 10.1097/PHM.0b013e31813e0de0

Massie, C. L., Kantak, S. S., Narayanan, P., and Wittenberg, G. F. (2015). Timing of motor cortical stimulation during planar robotic training differentially impacts neuroplasticity in older adults. Clin. Neurophysiol. 126, 1024-1032. doi: 10.1016/j.clinph.2014.06.053

Massie, C. L., Tracy, B. L., and Malcolm, M. P. (2013). Functional repetitive transcranial magnetic stimulation increases motor cortex excitability in survivors of stroke. Clin. Neurophysiol. 124, 371-378. doi: 10.1016/j.clinph.2012.07.026

Meehan, S. K., Dao, E., Linsdell, M. A., and Boyd, L. A. (2011). Continuous theta burst stimulation over the contralesional sensory and motor cortex enhances motor learning post-stroke. Neurosci. Lett. 500, 26-30. doi: 10.1016/j.neulet.2011.05.237

Meilink, A., Hemmen, B., Seelen, H. A., and Kwakkel, G. (2008). Impact of EMG-triggered neuromuscular stimulation of the wrist and finger extensors of the paretic hand after stroke: a systematic review of the literature. Clin. Rehabil. 22, 291-305. doi: 10.1177/026921550 7083368

Mihara, M., Hattori, N., Hatakenaka, M., Yagura, H., Kawano, T., Hino, T., et al. (2013). Near-infrared spectroscopy-mediated neurofeedback enhances efficacy of motor imagery-based training in poststroke victims: a pilot study. Stroke 44, 1091-1098. doi: 10.1161/STROKEAHA.111.674507

Motta-Oishi, A. A., Magalhaes, F. H., and Micolis De Azevedo, F. (2013). Neuromuscular electrical stimulation for stroke rehabilitation: is spinal plasticity a possible mechanism associated with diminished spasticity? Med. Hypotheses 81, 784-788. doi: 10.1016/j.mehy.2013.08.013

Muellbacher, W., Ziemann, U., Boroojerdi, B., Cohen, L., and Hallett, M. (2001). Role of the human motor cortex in rapid motor learning. Exp. Brain Res. 136, 431-438. doi: 10.1007/s002210000614

Muller, J. F., Orekhov, Y., Liu, Y., and Ziemann, U. (2007). Homeostatic plasticity in human motor cortex demonstrated by two consecutive sessions of paired associative stimulation. Eur. J. Neurosci. 25, 3461-3468. doi: 10.1111/j.14609568.2007.05603.x 
Muller-Dahlhaus, F., Ziemann, U., and Classen, J. (2010). Plasticity resembling spike-timing dependent synaptic plasticity: the evidence in human cortex. Front. Synaptic Neurosci. 2:34. doi: 10.3389/fnsyn.2010.00034

Murakami, T., Muller-Dahlhaus, F., Lu, M. K., and Ziemann, U. (2012). Homeostatic metaplasticity of corticospinal excitatory and intracortical inhibitory neural circuits in human motor cortex. J. Physiol. 590, 5765-5781. doi: 10.1113/jphysiol.2012.238519

Murphy, T. H., and Corbett, D. (2009). Plasticity during stroke recovery: from synapse to behaviour. Nat. Rev. Neurosci. 10, 861-872. doi: 10.1038/nrn2735

Nadeau, S. E., Behrman, A. L., Davis, S. E., Reid, K., Wu, S. S., Stidham, B. S., et al. (2004). Donepezil as an adjuvant to constraint-induced therapy for upper-limb dysfunction after stroke: an exploratory randomized clinical trial. J. Rehabil. Res. Dev. 41, 525-534. doi: 10.1682/JRRD.2003.07.0108

Nitsche, M. A., and Paulus, W. (2000). Excitability changes induced in the human motor cortex by weak transcranial direct current stimulation. J. Physiol. 527, 633-639. doi: 10.1111/j.1469-7793.2000.t01-1-00633.x

Nitsche, M. A., Roth, A., Kuo, M. F., Fischer, A. K., Liebetanz, D., Lang, N., et al. (2007). Timing-dependent modulation of associative plasticity by general network excitability in the human motor cortex. J. Neurosci. 27, 3807-3812. doi: 10.1523/JNEUROSCI.5348-06.2007

Nitsche, M. A., Schauenburg, A., Lang, N., Liebetanz, D., Exner, C., Paulus, W., et al. (2003). Facilitation of implicit motor learning by weak transcranial direct current stimulation of the primary motor cortex in the human. J. Cogn. Neurosci. 15, 619-626. doi: 10.1162/089892903321662994

Novak, D., Nagle, A., Keller, U., and Riener, R. (2014). Increasing motivation in robot-aided arm rehabilitation with competitive and cooperative gameplay. J. Neuroeng. Rehabil. 11:64. doi: 10.1186/1743-0003-11-64

Ochi, M., Saeki, S., Oda, T., Matsushima, Y., and Hachisuka, K. (2013). Effects of anodal and cathodal transcranial direct current stimulation combined with robotic therapy on severely affected arms in chronic stroke patients. J. Rehabil. Med. 45, 137-140. doi: 10.2340/16501977-1099

Page, S. J., Levine, P., and Khoury, J. C. (2009). Modified constraint-induced therapy combined with mental practice: thinking through better motor outcomes. Stroke 40, 551-554. doi: 10.1161/STROKEAHA.108.528760

Pascual-Leone, A., Valls-Sole, J., Wassermann, E. M., and Hallett, M. (1994). Responses to rapid-rate transcranial magnetic stimulation of the human motor cortex. Brain 117, 847-858. doi: 10.1093/brain/117.4.847

Pennati, G. V., Da Re, C., Messineo, I., and Bonaiuti, D. (2014). How the robotic training and the botolinum toxin could be combined in chronic post stroke upper limb spasticity? A pilot study. Eur. J. Phys. Rehabil. Med. [Epub ahead of print].

Pollock, A., Farmer, S. E., Brady, M. C., Langhorne, P., Mead, G. E., Mehrholz, J., et al. (2014). Interventions for improving upper limb function after stroke. Cochrane Database Syst. Rev. 11, CD010820. doi: 10.1002/14651858.CD010820.pub2

Popa, T., Velayudhan, B., Hubsch, C., Pradeep, S., Roze, E., Vidailhet, M., et al. (2013). Cerebellar processing of sensory inputs primes motor cortex plasticity. Cereb. Cortex 23, 305-314. doi: 10.1093/cercor/bhs016

Potter-Nerger, M., Fischer, S., Mastroeni, C., Groppa, S., Deuschl, G., Volkmann, J., et al. (2009). Inducing homeostatic-like plasticity in human motor cortex through converging corticocortical inputs. J. Neurophysiol. 102, 3180-3190. doi: 10.1152/jn.91046.2008

Ragert, P., Camus, M., Vandermeeren, Y., Dimyan, M. A., and Cohen, L. G. (2009). Modulation of effects of intermittent theta burst stimulation applied over primary motor cortex (M1) by conditioning stimulation of the opposite M1. J. Neurophysiol. 102, 766-773. doi: 10.1152/jn.00274.2009

Ramos-Murguialday, A., Broetz, D., Rea, M., Laer, L., Yilmaz, O., Brasil, F. L., et al. (2013). Brain-machine interface in chronic stroke rehabilitation: a controlled study. Ann. Neurol. 74, 100-108. doi: 10.1002/ana.23879

Rebesco, J. M., Stevenson, I. H., Kording, K. P., Solla, S. A., and Miller, L. E. (2010). Rewiring neural interactions by micro-stimulation. Front. Syst. Neurosci. 4:39. doi: 10.3389/fnsys.2010.00039

Rehme, A. K., Eickhoff, S. B., Wang, L. E., Fink, G. R., and Grefkes, C. (2011). Dynamic causal modeling of cortical activity from the acute to the chronic stage after stroke. Neuroimage 55, 1147-1158. doi: 10.1016/j.neuroimage.2011.01.014

Reinkensmeyer, D. J., Wolbrecht, E. T., Chan, V., Chou, C., Cramer, S. C., and Bobrow, J. E. (2012). Comparison of three-dimensional, assist-asneeded robotic arm/hand movement training provided with Pneu-WREX to conventional tabletop therapy after chronic stroke. Am. J. Phys. Med. Rehabil. 91, S232-S241. doi: 10.1097/phm.0b013e31826bce79

Ridding, M. C., and Ziemann, U. (2010). Determinants of the induction of cortical plasticity by non-invasive brain stimulation in healthy subjects. J. Physiol. 588, 2291-2304. doi: 10.1113/jphysiol.2010.190314

Rodriguez-Fornells, A., Rojo, N., Amengual, J. L., Ripolles, P., Altenmuller, E., and Munte, T. F. (2012). The involvement of audio-motor coupling in the music-supported therapy applied to stroke patients. Ann. N.Y. Acad. Sci. 1252, 282-293. doi: 10.1111/j.1749-6632.2011.06425.x

Rosser, N., Heuschmann, P., Wersching, H., Breitenstein, C., Knecht, S., and Floel, A. (2008). Levodopa improves procedural motor learning in chronic stroke patients. Arch. Phys. Med. Rehabil. 89, 1633-1641. doi: 10.1016/j.apmr.2008.02.030

Rossi, C., Sallustio, F., Di Legge, S., Stanzione, P., and Koch, G. (2013). Transcranial direct current stimulation of the affected hemisphere does not accelerate recovery of acute stroke patients. Eur. J. Neurol. 20, 202-204. doi: 10.1111/j.1468-1331.2012.03703.x

Sawaki, L., Yaseen, Z., Kopylev, L., and Cohen, L. G. (2003). Age-dependent changes in the ability to encode a novel elementary motor memory. Ann. Neurol. 53, 521-524. doi: 10.1002/ana.10529

Schulz, R., Braass, H., Liuzzi, G., Hoerniss, V., Lechner, P., Gerloff, C., et al. (2015). White matter integrity of premotor-motor connections is associated with motor output in chronic stroke patients. Neuroimage Clin. 7, 82-86. doi: 10.1016/j.nicl.2014.11.006

Schuster, C., Maunz, G., Lutz, K., Kischka, U., Sturzenegger, R., and Ettlin, T. (2011). Dexamphetamine improves upper extremity outcome during rehabilitation after stroke: a pilot randomized controlled trial. Neurorehabil. Neural Repair 25, 749-755. doi: 10.1177/1545968311405674

Seniow, J., Bilik, M., Lesniak, M., Waldowski, K., Iwanski, S., and Czlonkowska, A. (2012). Transcranial magnetic stimulation combined with physiotherapy in rehabilitation of poststroke hemiparesis: a randomized, double-blind, placebo-controlled study. Neurorehabil. Neural Repair 26, 1072-1079. doi: $10.1177 / 1545968312445635$

Shams, L., and Seitz, A. R. (2008). Benefits of multisensory learning. Trends Cogn. Sci. 12, 411-417. doi: 10.1016/j.tics.2008.07.006

Shin, H. K., Cho, S. H., Jeon, H. S., Lee, Y. H., Song, J. C., Jang, S. H., et al. (2008). Cortical effect and functional recovery by the electromyography-triggered neuromuscular stimulation in chronic stroke patients. Neurosci. Lett. 442, 174-179. doi: 10.1016/j.neulet.2008.07.026

Stagg, C. J., Bachtiar, V., O’Shea, J., Allman, C., Bosnell, R. A., Kischka, U., et al. (2012). Cortical activation changes underlying stimulationinduced behavioural gains in chronic stroke. Brain 135, 276-284. doi: 10.1093/brain/awr313

Stagg, C. J., Jayaram, G., Pastor, D., Kincses, Z. T., Matthews, P. M., and JohansenBerg, H. (2011). Polarity and timing-dependent effects of transcranial direct current stimulation in explicit motor learning. Neuropsychologia 49, 800-804. doi: 10.1016/j.neuropsychologia.2011.02.009

Starkey, M. L., and Schwab, M. E. (2014). How plastic is the brain after a stroke? Neuroscientist 20, 359-371. doi: 10.1177/1073858413514636

Stefan, K., Kunesch, E., Cohen, L. G., Benecke, R., and Classen, J. (2000). Induction of plasticity in the human motor cortex by paired associative stimulation. Brain 123, 572-584. doi: 10.1093/brain/123.3.572

Stinear, C. M., Barber, P. A., Smale, P. R., Coxon, J. P., Fleming, M. K., and Byblow, W. D. (2007). Functional potential in chronic stroke patients depends on corticospinal tract integrity. Brain 130, 170-180. doi: 10.1093/brain/ awl333

Stinear, C. M., and Byblow, W. D. (2014). Predicting and accelerating motor recovery after stroke. Curr. Opin. Neurol. 27, 624-630. doi: $10.1097 /$ wco.0000000000000153

Sun, S. F., Hsu, C. W., Sun, H. P., Hwang, C. W., Yang, C. L., and Wang, J. L. (2010). Combined botulinum toxin type A with modified constraint-induced movement therapy for chronic stroke patients with upper extremity spasticity: a randomized controlled study. Neurorehabil. Neural Repair 24, 34-41. doi: $10.1177 / 1545968309341060$

Sung, W. H., Wang, C. P., Chou, C. L., Chen, Y. C., Chang, Y. C., and Tsai, P. Y. (2013). Efficacy of coupling inhibitory and facilitatory repetitive transcranial magnetic stimulation to enhance motor recovery in hemiplegic stroke patients. Stroke 44, 1375-1382. doi: 10.1161/STROKEAHA.111.000522 
Takeuchi, N., Chuma, T., Matsuo, Y., Watanabe, I., and Ikoma, K. (2005). Repetitive transcranial magnetic stimulation of contralesional primary motor cortex improves hand function after stroke. Stroke 36, 2681-2686. doi: 10.1161/01.STR.0000189658.51972.34

Takeuchi, N., and Izumi, S. (2012a). Maladaptive plasticity for motor recovery after stroke: mechanisms and approaches. Neural Plast. 2012:359728. doi: $10.1155 / 2012 / 359728$

Takeuchi, N., and Izumi, S. (2012b). Noninvasive brain stimulation for motor recovery after stroke: mechanisms and future views. Stroke Res. Treat. 2012, 584727. doi: 10.1155/2012/584727

Takeuchi, N., Tada, T., Toshima, M., Chuma, T., Matsuo, Y., and Ikoma, K. (2008). Inhibition of the unaffected motor cortex by $1 \mathrm{~Hz}$ repetitive transcranical magnetic stimulation enhances motor performance and training effect of the paretic hand in patients with chronic stroke. J. Rehabil. Med. 40, 298-303. doi: 10.2340/16501977-0181

Takeuchi, N., Tada, T., Toshima, M., Matsuo, Y., and Ikoma, K. (2009). Repetitive transcranial magnetic stimulation over bilateral hemispheres enhances motor function and training effect of paretic hand in patients after stroke. J. Rehabil. Med. 41, 1049-1054. doi: 10.2340/16501977-0454

Taylor, J. L., and Martin, P. G. (2009). Voluntary motor output is altered by spiketiming-dependent changes in the human corticospinal pathway. J. Neurosci. 29, 11708-11716. doi: 10.1523/JNEUROSCI.2217-09.2009

Teo, J. T., Swayne, O. B., Cheeran, B., Greenwood, R. J., and Rothwell, J. C. (2011). Human theta burst stimulation enhances subsequent motor learning and increases performance variability. Cereb. Cortex 21, 1627-1638. doi: 10.1093/cercor/bhq231

Thabit, M. N., Ueki, Y., Koganemaru, S., Fawi, G., Fukuyama, H., and Mima, T. (2010). Movement-related cortical stimulation can induce human motor plasticity. J. Neurosci. 30, 11529-11536. doi: 10.1523/JNEUROSCI.182910.2010

Theilig, S., Podubecka, J., Bosl, K., Wiederer, R., and Nowak, D. A. (2011). Functional neuromuscular stimulation to improve severe hand dysfunction after stroke: does inhibitory rTMS enhance therapeutic efficiency? Exp. Neurol. 230, 149-155. doi: 10.1016/j.expneurol.2011. 04.010

Turrigiano, G. G. (2008). The self-tuning neuron: synaptic scaling of excitatory synapses. Cell 135, 422-435. doi: 10.1016/j.cell.2008.10.008

Veerbeek, J. M., van Wegen, E., van Peppen, R., van der Wees, P. J., Hendriks, E., Rietberg, M., et al. (2014). What is the evidence for physical therapy poststroke? A systematic review and meta-analysis. PLoS ONE 9:e87987. doi: 10.1371/journal.pone.0087987

Wang, C. P., Tsai, P. Y., Yang, T. F., Yang, K. Y., and Wang, C. C. (2014a). Differential effect of conditioning sequences in coupling inhibitory/facilitatory repetitive transcranial magnetic stimulation for poststroke motor recovery. CNS Neurosci. Ther. 20, 355-363. doi: 10.1111/cns.12221

Wang, Q. M., Cui, H., Han, S. J., Black-Schaffer, R., Volz, M. S., Lee, Y. T., et al. (2014b). Combination of transcranial direct current stimulation and methylphenidate in subacute stroke. Neurosci. Lett. 569, 6-11. doi: 10.1016/j.neulet.2014.03.011

Westlake, K. P., Hinkley, L. B., Bucci, M., Guggisberg, A. G., Byl, N., Findlay, A. M., et al. (2012). Resting state alpha-band functional connectivity and recovery after stroke. Exp. Neurol. 237, 160-169. doi: 10.1016/j.expneurol.2012.06.020

Wolters, A., Sandbrink, F., Schlottmann, A., Kunesch, E., Stefan, K., Cohen, L. G., et al. (2003). A temporally asymmetric Hebbian rule governing plasticity in the human motor cortex. J. Neurophysiol. 89, 2339-2345. doi: 10.1152/jn.00900.2002

Yamada, N., Kakuda, W., Kondo, T., Mitani, S., Shimizu, M., and Abo, M. (2014). Local muscle injection of botulinum toxin type a synergistically improves the beneficial effects of repetitive transcranial magnetic stimulation and intensive occupational therapy in post-stroke patients with spastic upper limb hemiparesis. Eur. Neurol. 72, 290-298. doi: 10.1159/000365005

Yoon, J. A., Koo, B. I., Shin, M. J., Shin, Y. B., Ko, H. Y., and Shin, Y. I. (2014). Effect of constraint-induced movement therapy and mirror therapy for patients with subacute stroke. Ann. Rehabil. Med. 38, 458-466. doi: 10.5535/arm.2014.38.4.458

Zheng, J., Liu, L., Xue, X., Li, H., Wang, S., Cao, Y., et al. (2013). Cortical electrical stimulation promotes neuronal plasticity in the peri-ischemic cortex and contralesional anterior horn of cervical spinal cord in a rat model of focal cerebral ischemia. Brain Res. 1504, 25-34. doi: 10.1016/j.brainres.2013.01.015

Zhu, L. L., Lindenberg, R., Alexander, M. P., and Schlaug, G. (2010). Lesion load of the corticospinal tract predicts motor impairment in chronic stroke. Stroke 41, 910-915. doi: 10.1161/STROKEAHA.109.577023

Ziemann, U., Ilic, T. V., Pauli, C., Meintzschel, F., and Ruge, D. (2004). Learning modifies subsequent induction of long-term potentiation-like and long-term depression-like plasticity in human motor cortex. J. Neurosci. 24, 1666-1672. doi: 10.1523/JNEUROSCI.5016-03.2004

Zimerman, M., Heise, K. F., Hoppe, J., Cohen, L. G., Gerloff, C., and Hummel, F. C. (2012). Modulation of training by single-session transcranial direct current stimulation to the intact motor cortex enhances motor skill acquisition of the paretic hand. Stroke 43, 2185-2191. doi: 10.1161/STROKEAHA.111.645382

Zimerman, M., Nitsch, M., Giraux, P., Gerloff, C., Cohen, L. G., and Hummel, F. C. (2013). Neuroenhancement of the aging brain: restoring skill acquisition in old subjects. Ann. Neurol. 73, 10-15. doi: 10.1002/ana.23761

Conflict of Interest Statement: The authors declare that the research was conducted in the absence of any commercial or financial relationships that could be construed as a potential conflict of interest.

Copyright (C) 2015 Takeuchi and Izumi. This is an open-access article distributed under the terms of the Creative Commons Attribution License (CC BY). The use, distribution or reproduction in other forums is permitted, provided the original author(s) or licensor are credited and that the original publication in this journal is cited, in accordance with accepted academic practice. No use, distribution or reproduction is permitted which does not comply with these terms. 